\title{
Hyperuricemia predicts poor prognosis of patients with cervical cancer
}

\author{
Cuihong Li ${ }^{1}$, Yufeng $\mathrm{Li}^{2}$, Na Su${ }^{2}$, Qiongna Liu ${ }^{3}$ and Chunxiang Chai ${ }^{4}$ \\ ${ }^{1}$ Department of Gynaecology and Obstetrics, Yidu Central Hospital of Weifang, Weifang 261000, China \\ 2 Department of Oncology, People's Hospital of Rizhao, Rizhao 276800, China \\ ${ }^{3}$ Department of Obstetrics and Gynecology, Huai'an Second People's Hospital and The Affiliated Huai'an Hospital of Xuzhou \\ Medical University, Huai'an 223001, China \\ ${ }^{4}$ Department of Rheumatology and Immunology, The Affiliated Hospital of Weifang Medical University, Weifang 261000, China \\ Correspondence to: Chunxiang Chai, email: wyfychai@sina.com \\ Yufeng Li, email: liyufeng0713@163.com
}

Keywords: cervical cancer; uric acid; hyperuricemia; prognosis

Received: August 18, $2017 \quad$ Accepted: December 11,2017 Published: January 02, 2018

Copyright: Li et al. This is an open-access article distributed under the terms of the Creative Commons Attribution License 3.0 (CC BY 3.0), which permits unrestricted use, distribution, and reproduction in any medium, provided the original author and source are credited.

\section{ABSTRACT}

Previous studies have suggested that hyperuricemia plays an important role in cancer initiation and progression; however, little is known about the role of hyperuricemia in the prognosis of cervical cancer. The aim of this study was to evaluate the association between hyperuricemia and cervical cancer by conducting a retrospective cohort study. 50 cervical cancer patients with hyperuricemia were randomly selected and 1:1 age-matched to 50 normouricemia patients with cervical cancer. We used the Kaplan-Meier survival analysis to determine the overall survival of patients with cervical cancer. Cox regression analysis was also adopted to estimate the role of hyperuricemia in cervical cancer prognosis primarily adjusted by age, tumor stage, status of histological grade, lymph node involvement, and histopathological subtype. The Kaplan-Meier analysis showed patients with hyperuricemia had poorer overall survival than those with normouricemia $(P=0.0086)$. The univariate and multivariate Cox analyses both showed that hyperuricemia was negatively associated with the overall survival of patients with cervical cancer. Our study suggests that hyperuricemia is related to poor prognosis of patients with cervical cancer.

\section{INTRODUCTION}

To the best of our knowledge, cervical cancer is a common cause of cancer-related deaths in females. Papanicolaou test and adjunct human papillomavirus testing can help to early screen for this cancer and make cervical cancer a treatable disease [1,2]. During the past few decades, metabolism-related factors have been suggested in tumorigenesis and tumor prognosis, including hyperuricemia [3-5]. Pathways involved in metabolism can be targeted for therapeutic purposes. Hyperuricemia is caused by abnormal metabolism of uric acid, which has been demonstrated to be involved in the pathogenesis of gout, cardiovascular diseases, respiratory diseases and tumors [4, 6-8]. Although serum uric acid possesses antioxidant properties, increased levels of serum uric acid can lead to local or systematic inflammatory response in human body and thus promote the occurrence of tumors.

It has been well established that the state of chronic and sustained inflammation is closely related to the cervical carcinogenesis and tumor biology [9-11]. Thus, hyperuricemia may confer effects on the initiation and progression of cervical cancer. However, little is known of the prognostic role of hyperuricemia in this deadly disease. In the current study, we aimed to investigate the association between hyperuricemia and prognosis of cervical cancer patients. In addition, we intended to elucidate the potential effect of hyperuricemia in combination with other factors correlated with the prognosis of cervical cancer patients, such as tumor stage and lymph node involvement. 


\section{RESULTS}

\section{Characteristics of all patients}

Patients' characteristics are provided in Table 1. 50 cervical cancer patients with hyperuricemia were randomly selected and matched to 50 cervical cancer patients with normouricemia by age. No significant discrepancies were found between the two groups with regard to mean age, histopathological type, tumor stage, differentiation grade, lymph node involvement, and median time of follow-up.

\section{Association between levels of serum uric acid and clinicopathological factors of cervical cancer}

Levels of serum uric acid did not alter with the tumor stage, histological grade, lymph node involvement as well as histopathological type of patients with cervical cancer (Figure 1).

\section{High levels of uric acid promoted the pro- liferation of HeLa cells}

MTT assay revealed that the proliferation of $\mathrm{HeLa}$ cells was significantly promoted in a time-dependent manner when stimulated with uric acid (Figure 2). Experiments were repeated for three times.

\section{Hyperuricemia related to poor overall survival of patients with cervical cancer}

As suggested by the Kaplan-Meier analysis, the overall survival of cervical cancer patients with hyperuricemia was poor in contrast to those patients with normouricemia with a $P$ value for log-rank test equal to 0.0086 (Figure 3). As evidenced by the analysis based on univariate Cox regression, cervical cancer patients with hyperuricemia had shorter overall survival than patients with normouricemia (hazard risk $(\mathrm{HR})=3.47,95 \%$ confidence interval (CI) 1.29-9.35) (Table 2). When the relationship of survival of cervical cancer patients with hyperuricemia was estimated by the multivariate Cox analysis, it was revealed that hyperuricemia might be an independent prognostic factor for cervical cancer patients adjusting by age, stage, status of histological grade, lymph node involvement, and histopathological type $(\mathrm{HR}=3.45$, 95\% CI 1.26-9.49) (Table 2). As shown in Table 2, hyperuricemia patients with advanced cervical cancer had poor prognosis compared to those with normouricemia.

\section{DISCUSSION}

The current study firstly shows strong evidence that hyperuricemia independently correlates with poor prognosis of patients with cervical cancer. Targeting uric acid metabolism may be a promising strategy for improving the prognosis of patients with cervical cancer.
It is well known that uric acid is the product of purine metabolism. Serum uric acid has direct immunemodulating activity and is involved in the process of inflammatory response. Currently available studies have implicated that high levels of blood uric acid may break out homeostasis and increase the risk of a number of cardiovascular diseases [12]. Additionally, cumulative evidence has suggested that hyperuricemia is also closely related to metabolic syndrome, renal diseases, cancers, and other inflammation-related diseases [12-15]. Hyperuricemia can induce the generation of inflammatory mediators, for instance, TNF- $\alpha$, IL- 6 , and MCP-1, by activating NF- $\mathrm{\kappa B}$ signaling pathway [16-18]. Considerable data have shown that inflammation is highly associated with the carcinogenesis and cancer progression [19-21]. Therefore, hyperuricemia-related inflammation may confer effects on the development and progression of malignant tumors. During the past few years, the association of hyperuricemia with cancer prognosis has drawn much attention. Accumulated data have shown strong evidence that hyperuricemia is related to higher risk of cancer incidence and mortality [14, 22, 23]. Nevertheless, little is known about the modifying effects of serum uric acid-induced inflammation on the prognosis of cervical cancer patients. The present study, for the first time, shows strong evidence that high levels of uric acid can promote the proliferation of cervical cancer cells, suggesting that uric acid may be a risk factor for this deadly disease. Nonetheless, the underlying molecular mechanisms regarding uric acid metabolism in cervical carcinogenesis remain largely unknown. In addition, we find that hyperuricemia is a critical prognostic factor for cervical cancer patients. Cervical cancer patients with hyperuricemia have poorer prognosis than those with normouricemia.

Hyperuricemia not only alters the risk of cancer, but modifies the prognosis of a number of cancers, including digestive organs, respiratory system and intrathoracic organs $[14,24]$. Uric acid is a potent antioxidant and thus may protect individuals against malignant tumors and predict good prognosis of diseases. However, few studies have demonstrated the negative relationship between uric acid in serum and malignant tumors. Furthermore, previous findings are inconsistent and inconclusive $[23,25,26]$. Strasak AM and the colleagues have found that hyperuricemia is independently related to elevated risk of cancer mortality [25]. Increased risk of death from cardiovascular diseases is also demonstrated to be related to high levels of serum uric acid [27]. Taken together, serum uric acid may confer bad effects on the prognosis of several diseases, including cancer. Similar to the findings mentioned above, findings in the present study are inconsistent with the proposed antioxidant and protective effects of serum uric acid against cancer. It is strongly suggested that hyperuricemia correlates with poor overall survival of cervical cancer patients, which reflects 
Table 1: Characteristics of all cervical cancer patients

\begin{tabular}{|c|c|c|c|}
\hline Parameter & Hyperuricemia $(n=50)$ & Normouricemia $(n=50)$ & $p$-value \\
\hline $\begin{array}{l}\text { Mean age in years at first } \\
\text { diagnosis (Mean } \pm \text { SD) }\end{array}$ & $48.8 \pm 10.8$ & $49.7 \pm 12.8$ & 0.70 \\
\hline Age, $n(\%)$ & & & 0.84 \\
\hline$<49$ years & $24(48.0)$ & $25(50.0)$ & \\
\hline$\geq 49$ years & $26(52.0)$ & $25(50.0)$ & \\
\hline Histopathological type, $n(\%)$ & & & 0.84 \\
\hline Squamous cell carcinoma & $30(60.0)$ & $31(62.0)$ & \\
\hline Adenocarcinoma & $17(34.0)$ & $16(32.0)$ & \\
\hline Adenosquamous carcinoma & $3(6.0)$ & $3(6.0)$ & \\
\hline Tumor stage, $n(\%)$ & & & 0.38 \\
\hline $\mathrm{I} / \mathrm{II}$ & $42(84.0)$ & $45(90.0)$ & \\
\hline III/IV & $8(16.0)$ & $5(10.0)$ & \\
\hline Differentiation grade, $n(\%)$ & & & 0.46 \\
\hline G1 & $7(14.0)$ & $7(14.0)$ & \\
\hline G2 & $31(62.0)$ & $34(68.0)$ & \\
\hline G3 & $12(24.0)$ & $9(18.0)$ & \\
\hline Lymph node involvement, $n$ (\%) & & & 0.48 \\
\hline Positive & $13(26.0)$ & $10(20.0)$ & \\
\hline Negative & $37(74.0)$ & $40(80.0)$ & \\
\hline $\begin{array}{l}\text { Median time of follow-up } \\
\text { (months, Mean } \pm \text { SD) }\end{array}$ & $46.1 \pm 11.8$ & $45.9 \pm 12.0$ & 0.93 \\
\hline
\end{tabular}
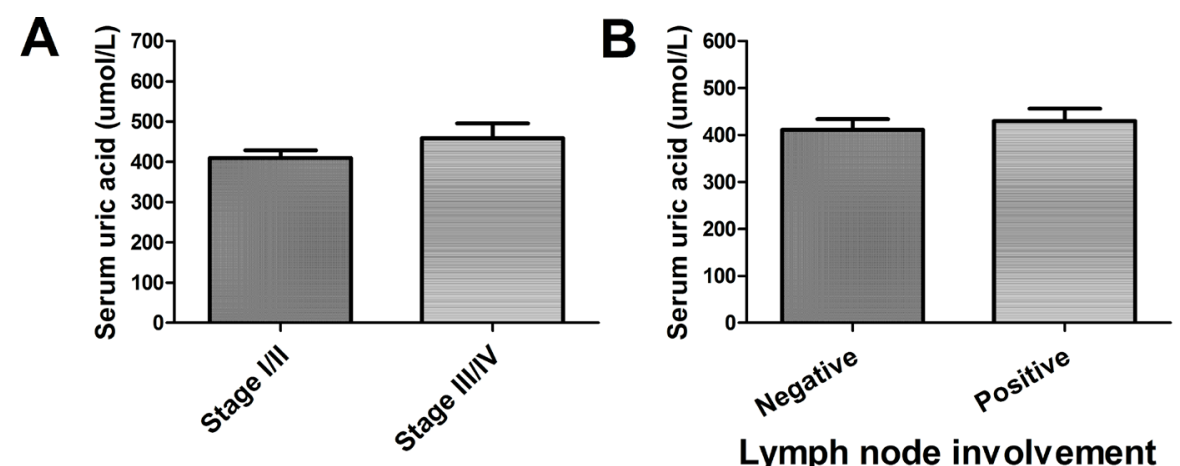

\section{Lymph node involvement}
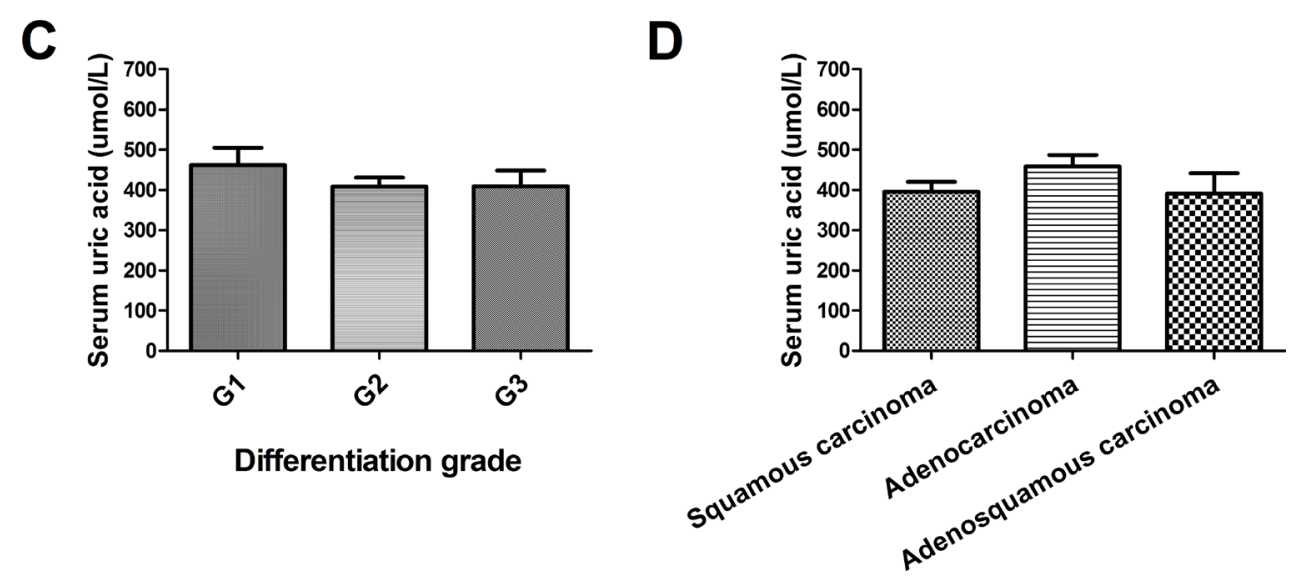

Figure 1: Correlation between serum uric acid and pathological factors (A) Association between levels of serum uric acid and tumor stage of cervical cancer patients; (B) Association between levels of serum uric acid and lymph node involvement of cervical cancer patients; (C) Association between levels of serum uric acid and tumor differentiation grade of cervical cancer patients; (D) Association between levels of serum uric acid and pathological types of cervical cancer patients. 
more serious prognosis with regard to high levels of uric acid in serum.

There is a potential limitation in this study, the sample size of which is relatively small. More future studies with sufficient statistical power are warranted to provide strong evidence for the potential role of uric acid in serum associated with the prognosis of cervical cancer patients. In addition, another limitation of this study is brief duration of follow-up time when investigating the association of hyperuricemia with cancer prognosis, which may lead to random or systematic errors. More relevant studies with high quality are encouraged for further investigation.

In summary, our study firstly provides new insight into the role of hyperuricemia in the prognosis of patients with cervical cancer. The prognosis of cervical cancer patients with hyperuricemia is much poorer than those with normouricemia. However, more relevant studies with large sample size are warranted for further elucidation.

\section{MATERIALS AND METHODS}

\section{Patients}

In the present study, a total of 100 patients with histologically diagnosed cervical cancer (from 2006 to 2011) treated in our hospital were enrolled. Among them, 50 cervical cancer patients were subject to with hyperuricemia, while the other 50 patients with normouricemia were 1:1 matched to patients with hyperuricemia by age. The mean

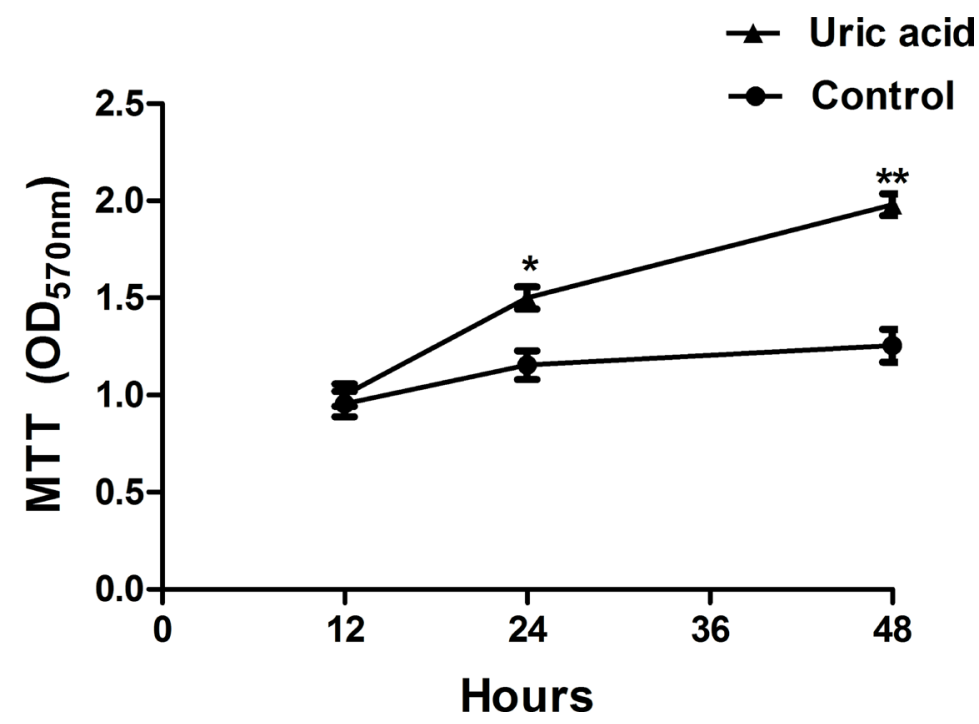

Figure 2: MTT assay for the proliferation of HeLa cells $\left(n=3 ;{ }^{*} P<0.05 ;{ }^{* *} P<0.01\right)$.

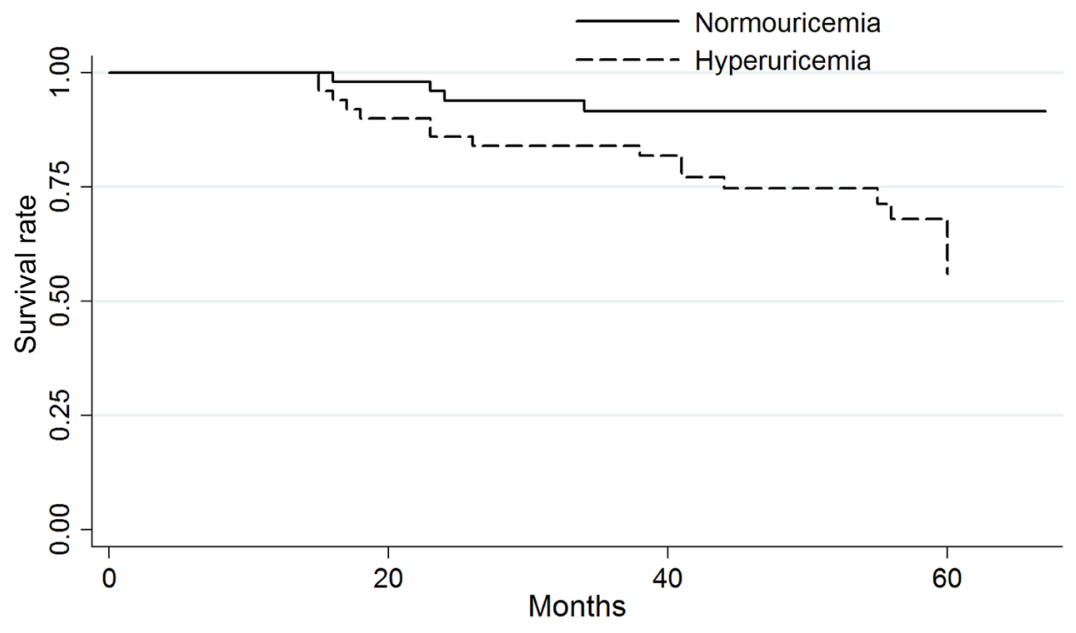

Kaplan-Meier survival estimates

Figure 3: Kaplan-Meier curve analysis for the relationship between hyperuricemia and prognosis of cervical cancer patients $(P$ value for log-rank test $=\mathbf{0 . 0 0 8 6})$. 
Table 2: Cox regression analysis for the association of hyperuricemia with cervical cancer prognosis

\begin{tabular}{|c|c|c|c|c|}
\hline \multirow{2}{*}{ Factors } & \multicolumn{2}{|c|}{ Univariate analysis } & \multicolumn{2}{|c|}{ Multivariate analysis } \\
\hline & HR $(95 \% \mathrm{CI})$ & $p$-value & HR $(95 \% \mathrm{CI})$ & $p$-value \\
\hline Age ( $\geq 49$ years vs. $<49$ years) & $1.18(0.52-2.69)$ & 0.697 & $0.95(0.40-2.26)$ & 0.90 \\
\hline Stage (III/IV vs. I/II) & $3.00(1.31-6.85)$ & 0.009 & $2.45(1.12-5.92)$ & 0.046 \\
\hline Histological grade (G3 vs. G1/G2) & $0.60(0.18-2.03)$ & 0.413 & $0.41(0.12-1.44)$ & 0.164 \\
\hline $\begin{array}{l}\text { Lymph node involvement (Positive } \\
\text { vs. Negative) }\end{array}$ & $2.67(1.17-6.08)$ & 0.020 & $2.75(1.20-6.32)$ & 0.017 \\
\hline $\begin{array}{l}\text { Histopathological type (Squamous } \\
\text { cell carcinoma vs. Adenocarcinoma) }\end{array}$ & $0.60(0.26-1.38)$ & 0.234 & $0.55(0.23-1.30)$ & 0.174 \\
\hline Hyperuricemia & $3.47(1.29-9.35)$ & 0.014 & $3.45(1.26-9.49)$ & 0.016 \\
\hline
\end{tabular}

level of serum uric acid of all cervical cancer patients was $435.56 \pm 34.51 \mathrm{umol} / \mathrm{L}$. All patients had signed the informed consent prior to study inclusion. The current study was approved by the hospital's Institutional Ethics Committee.

\section{Uric acid assay}

Blood samples were acquired by puncturing from peripheral venous $24-48 \mathrm{~h}$ prior to clinical treatment during routine preoperative assessment, and were used for evaluation of levels of uric acid in serum samples from patients with cervical cancer. Serum uric acid was assayed by way of EDTA hydrazine on the autoanalyzer based on the basic protocols [28]. Levels of serum uric acid $>420$ umol/L was defined as hyperuricemia.

\section{MTT assay}

We used the cell growth determination kit MTT based (Sigma, USA) to estimate the proliferation of HeLa cells stimulated with uric acid $(16 \mathrm{mg} / \mathrm{dL}) .2 \times 10^{5} \mathrm{HeLa}$ cells were cultured in RPMI 1640 medium (Invitrogen, USA) supplemented with penicillin $(100 \mathrm{U} / \mathrm{ml})$ and streptomycin (100 mg/ml) (Invitrogen, USA) for 12, 24 and 48 hours. After incubated with MTT solution for 4 hours, the absorption $(570 \mathrm{~nm})$ was determined by use of the microplate reader (Bio-Tek, USA).

\section{Outcomes estimation}

The association between hyperuricemia and cervical cancer outcome was investigated by overall survival estimation. In this study, overall survival was established as from time of making the definite diagnosis or accepting surgery to death or the final follow-up time. We carried out the follow-up every six months up till to loss to follow-up, death, or follow-up for a total of 5 years. The latest followup time in this study was conducted in March, 2016.

\section{Statistical analysis}

The association between hyperuricemia and the overall survival of patients with cervical cancer was estimated by the Kaplan-Meier curve analysis. In addition, we used the log-rank test to assess the survival rate of cervical cancer patients. Cox regression analysis was used to determine the relative risk of mortality. The STATA (version 12.0) software was applied for all statistical analysis, and $P<0.05$ was regarded to be statistically significant in this research.

\section{CONFLICTS OF INTEREST}

The authors declare no conflicts of interests.

\section{FUNDING}

This work was supported by grants from the Medical and Health Science and Technology Development Program of Shandong Province (2016WS0681).

\section{REFERENCES}

1. Wuerthner BA, Avila-Wallace M. Cervical cancer: Screening, management, and prevention. Nurse Pract. 2016; 41:18-23.

2. Saraiya M, Steben M, Watson M, Markowitz L. Evolution of cervical cancer screening and prevention in United States and Canada: implications for public health practitioners and clinicians. Prev Med. 2013; 57:426-433.

3. Ara SA, Ashraf S, Patil BM. Evaluation of serum uric acid levels in patients with oral squamous cell carcinoma. Indian J Dent Res. 2016; 27:178-183.

4. Wang S, Liu X, He Z, Chen X, Li W. Hyperuricemia has an adverse impact on the prognosis of patients with osteosarcoma. Tumour Biol. 2016; 37:1205-1210.

5. Altman BJ, Stine ZE, Dang CV. From Krebs to clinic: glutamine metabolism to cancer therapy. Nat Rev Cancer. $2016 ; 16: 773$.

6. Abrahams MN. Gout and hyperuricaemia. S Afr Med J. 2015; 105:1078.

7. Hamaguchi S, Furumoto T, Tsuchihashi-Makaya M, Goto K, Goto D, Yokota T, Kinugawa S, Yokoshiki H, Takeshita A, Tsutsui H. Hyperuricemia predicts adverse outcomes in patients with heart failure. Int J Cardiol. 2011; 151:143-147. 
8. Zhang $\mathrm{X}$, Liu L, Liang $\mathrm{R}$, Jin S. Hyperuricemia is a biomarker of early mortality in patients with chronic obstructive pulmonary disease. Int J Chron Obstruct Pulmon Dis. 2015; 10:2519-2523.

9. Sommer P, Ray DW. Novel therapeutic agents targeting the glucocorticoid receptor for inflammation and cancer. Curr Opin Investig Drugs. 2008; 9:1070-1077.

10. Qiao L, Li X. Role of chronic inflammation in cancers of the gastrointestinal system and the liver: where we are now. Cancer Lett. 2014; 345:150-152.

11. Wu Y, Antony S, Meitzler JL, Doroshow JH. Molecular mechanisms underlying chronic inflammation-associated cancers. Cancer Lett. 2014; 345:164-173.

12. Jalal DI. Hyperuricemia, the Kidneys, and the Spectrum of Associated Diseases: A Narrative Review. Curr Med Res Opin. 2016:1-26.

13. Bonakdaran S, Kharaqani B. Association of serum uric acid and metabolic syndrome in type 2 diabetes. Curr Diabetes Rev. 2014; 10:113-117.

14. Yan S, Zhang P, Xu W, Liu Y, Wang B, Jiang T, Hua C, Wang X, Xu D, Sun B. Serum Uric Acid Increases Risk of Cancer Incidence and Mortality: A Systematic Review and Meta-Analysis. Mediators Inflamm. 2015; 2015:764250.

15. Wang W, Xu D, Wang B, Yan S, Wang X, Yin Y, Wang X, Sun B, Sun X. Increased Risk of Cancer in relation to Gout: A Review of Three Prospective Cohort Studies with 50,358 Subjects. Mediators Inflamm. 2015; 2015:680853.

16. Lu W, Xu Y, Shao X, Gao F, Li Y, Hu J, Zuo Z, Shao $\mathrm{X}$, Zhou L, Zhao Y, Cen X. Uric Acid Produces an Inflammatory Response through Activation of NF-kappaB in the Hypothalamus: Implications for the Pathogenesis of Metabolic Disorders. Sci Rep. 2015; 5:12144.

17. Sharon Y, Schlesinger N. Beyond Joints: a Review of Ocular Abnormalities in Gout and Hyperuricemia. Curr Rheumatol Rep. 2016; 18:37.

18. Xie H, Sun J, Chen Y, Zong M, Li S, Wang Y. EGCG Attenuates Uric Acid-Induced Inflammatory and Oxidative Stress Responses by Medicating the NOTCH Pathway. Oxid Med Cell Longev. 2015; 2015:214836.
19. Marx J. Cancer research. Inflammation and cancer: the link grows stronger. Science. 2004; 306:966-968.

20. Sun B, Karin M. Inflammation and liver tumorigenesis. Front Med. 2013; 7:242-254.

21. Sun B, Karin M. The therapeutic value of targeting inflammation in gastrointestinal cancers. Trends Pharmacol Sci. 2014; 35:349-357.

22. Chen CJ, Yen JH, Chang SJ. Gout patients have an increased risk of developing most cancers, especially urological cancers. Scand J Rheumatol. 2014; 43:385-390.

23. Boffetta P, Nordenvall C, Nyren O, Ye W. A prospective study of gout and cancer. Eur J Cancer Prev. 2009; 18:127-132.

24. Strasak AM, Rapp K, Hilbe W, Oberaigner W, Ruttmann E, Concin H, Diem G, Pfeiffer KP, Ulmer H. Serum uric acid and risk of cancer mortality in a large prospective male cohort. Cancer Causes Control. 2007; 18:1021-1029.

25. Strasak AM, Rapp K, Hilbe W, Oberaigner W, Ruttmann E, Concin H, Diem G, Pfeiffer KP, Ulmer H. The role of serum uric acid as an antioxidant protecting against cancer: prospective study in more than 28000 older Austrian women. Ann Oncol. 2007; 18:1893-1897.

26. Ames BN, Cathcart R, Schwiers E, Hochstein P. Uric acid provides an antioxidant defense in humans against oxidantand radical-caused aging and cancer: a hypothesis. Proc Natl Acad Sci U S A. 1981; 78:6858-6862.

27. Strasak AM, Kelleher CC, Brant LJ, Rapp K, Ruttmann E, Concin H, Diem G, Pfeiffer KP, Ulmer H. Serum uric acid is an independent predictor for all major forms of cardiovascular death in 28,613 elderly women: a prospective 21-year follow-up study. Int J Cardiol. 2008; 125:232-239.

28. Patel CP. Semimicro method for determination of serum uric acid using EDTA-hydrazine. Clin Chem. 1968; 14:764-775. 